\title{
Rapport annuel 2004 FMH Services
}

\section{FMH Services}

La gestion de notre société coopérative a connu quelques changements. En effet, suite au départ de notre directeur de longue date M. Markus Baumgartner vers le milieu de l'année 2004, notre groupe a été dirigé de façon intérimaire. Dans l'intervalle, nous avons pu engager M. Reinhard Kunz qui assumera la direction de FMH Services a partir du $1^{\text {er }}$ août 2005.

L'exercice 2004 a été marqué par la vente à la FMH (Fédération) de l'immeuble en copropriété de l'Elfenstrasse 18 à Berne, ce qui a généré un important bénéfice comptable. Cette situation favorable a permis de constituer une provision en vue d'éliminer le risque lié au cautionnement fourni en faveur d'un médecin en pratique privée, cautionnement dont on ne sait pas si le montant sera sollicité.

\section{FMH Consulting Services}

FMH Consulting Services et FMH Inkasso Services sont les piliers du réseau du groupe FMH-Services. Le conseil direct aux médecins est la tâche principale de FMH Consulting Services qui propose un produit complet englobant notamment des séminaires et des conseils personnalisés. Durant l'exercice écoulé, le groupe FMH Services a continué à se développer et à étendre son offre de prestations. En 2004, plus de 700 médecins ont participé aux séminaires sur des thèmes tels que l'ouverture, la remise ou la reprise d'un cabinet, la comptabilité et la fiscalité, l'informatique ainsi que la radiologie et le laboratoire au cabinet médical. Le nombre de participants a pu être augmenté par rapport à l'année précédente. Outre l'assistance lors de l'ouverture d'un nouveau cabinet, nous conseillons également les médecins en cas de reprise ou de remise d'une pratique. Les estimations de la valeur d'inventaire et de la valeur immatérielle («goodwill») d'un cabinet médical, basées sur des chiffres réalistes, restent un instrument très apprécié pour en déterminer la valeur de vente. Ces estimations ont été plus nombreuses que l'année précédente. Comme les médias s'en sont fait l'écho à plusieurs reprises, il devient plus difficile de vendre un cabinet médical. FMH Services continuera à tout mettre en œuvre pour assister les médecins dans ce domaine. Le réseau des fiduciaires regroupées dans FMH Services Treuhand, ainsi que le nombre de conseillers de FMH Insurance \& Financial Services ont été étendus au cours de l'exercice écoulé et de nouvelles succursales ont été ouvertes, en vue de pouvoir offrir nos conseils dans l'ensemble de la Suisse. Les produits de FMH Insurance \& Financial Services sont constamment adaptés au marché et exclusivement réservés à nos partenaires. Nous garantissons le professionnalisme des conseils par une procédure de sélection complexe et des cours de formation permanents. En effet, tous les conseillers de FMH Services se soumettent à une procédure de reconnaissance en plusieurs étapes durant laquelle ils doivent prouver qu'ils possèdent les qualifications requises. Cette procédure de sélection permet de garantir aux membres de la FMH des prestations de conseil de haute qualité.

Les annonces publiées dans la rubrique «Office de placement» ont subi un recul de $18 \%$. Cette baisse correspond à la tendance générale à la stagnation, voire à la régression, observée dans le volume global des annonces paraissant dans les journaux.

\section{FMH Inkasso Services}

Les Suisses ont la réputation de ne pas être des payeurs exemplaires. Il est de fait qu'au cours de l'année 2004, FMH Inkasso Services a traité plus de 21000 notes d'honoraires de médecins par voie pré-juridique ou juridique. Le taux de succès obtenu dans ce domaine par l'organisation de services du corps médical demeure très bon. La pression psychologique qui s'exerce sur le débiteur lorsqu'il voit le logo d'un bureau d'encaissement professionnel ne doit pas être sous-estimée: il sait ainsi clairement que l'affaire (la note d'honoraire impayée) n'a pas été simplement classée, mais qu'elle est traitée par des spécialistes de l'encaissement. Nous nous efforçons néanmoins de recouvrer les montants impayés dans le respect de la relation médecin-patient, sans employer la force et en cherchant une solution qui convienne tant au débiteur qu'au créditeur.

Dans l'ensemble, l'exercice 2004 s'est avéré très positif pour FMH Services et nous sommes heureux de continuer à servir et conseiller nos membres pendant la nouvelle année en cours. 


\section{Bilan au 31 décembre 2004}

\section{ACTIFS}

Capital de roulement

Avoir en banque

Titres et valeurs

Créances envers des organisations affiliées

Autres créances à court terme envers des filiales

Autres créances à court terme

Actifs de régularisation

\section{Capital investi}

Immeubles

Titres

Participations

Créances à long terme envers des organisations affiliées

Biens mobiliers

Total des actifs
Fr.

1054170.17

766705.42

237784.85

39155.00

5829

4695.70

5527994.00

0

1000.00

1696700.00

2499755.00

2601.00

5254226.17

\section{PASSIFS}

Capitaux étrangers

685246.90

15046.90

22000.00

200000.00

Autres dettes à court terme envers des organisation affiliées

300000.00

148200.00

Provisions à long terme

Capital effectif

Réserve générale

1418926.65

Total des passifs 


\title{
Compte d'exploitation du $1^{\text {er }}$ janvier au 31 décembre 2004
}

\author{
Recettes provenant de services \\ Activités de conseil \\ Revenus provenant de placements financiers \\ Placements financiers \\ Gain sur aliénation de placements \\ Revenus provenant de placements financiers, de services et d'aliénation de placements \\ Frais d'exploitation \\ Frais de personnel \\ 167118.45 \\ Autres frais d'exploitation \\ Gain d'exploitation avant amortissements et impôts \\ Impôts \\ Gain d'exploitation avant amortissements \\ Dissolution des provisions pour débiteurs \\ Formation de provisions pour cautionnement \\ Gain d'exploitation 1.1.2004-31.12. 2004

\section{Annexe}

Données facultatives selon l'art. 663b CO.

31. 12.2003

470000.00

369608.37

dont montant sollicité

1233493.00

2366300.00

865850.00

Biens mobiliers, total de la police d'assurance
31. 12.2004

470000.00

338140.79

\section{0}

0

865850.00

(police collective avec d'autres sociétés FMH)

La police d'assurance relative aux biens mobiliers couvre également les biens mobiliers

de FMH Inkasso Services et de FMH Consulting Services, lesquels sont ainsi compris

dans la valeur d'assurance-incendie.

\section{Rapport de l'organe de contrôle}

Après avoir procédé à la vérification des comptes annuels, l'organe de contrôle PriceWaterhouseCoopers recommande de les approuver en votation générale. 